\title{
Review of Christian List and Philip Pettit's Group agency: the possibility, design, and status of corporate agents. Oxford: Oxford University Press, 2011, 240 pp.
}

\section{CARLO MARTINI \\ University of Tilburg}

When thinking about the concept of 'group agency', a number of questions come to mind. We may wish to ask, in the first place, whether it is even legitimate to consider a group as an agent. Is it not just a linguistic shortcut to attribute knowledge, or even action, to a group, for example when we claim that a research centre has mapped the DNA of a new organism? The first and foremost task that List and Pettit undertake in the book being reviewed here is to explain why we should consider certain groups as agents. The authors defend the claim that some groups, just like individuals, satisfy all the conditions for being an agent. That is, to possess a world view, to have a set of plans or desires as to how the world should look like, and to have the capacity to bring actions about in order to change the world so that it looks (more) like those plans.

List and Pettit defend a version of non-reductionism for group agents that does not imply the existence of emergent properties of groups over and above those of the individuals. The problem of whether groups can be considered autonomous agents is not new, but the major contribution of this book is in bringing together a number of very powerful analytic tools (among others, social choice theory and Bayesian epistemology), as well as concepts from philosophy of mind and moral theory, which allow the authors to tackle some of the most pressing questions on group agency. The authors intend the book as a "pilot project" (p. 16) insofar as it shows dead ends as well as open paths for the research on group agents to follow.

List and Pettit start the discussion with a bird's eye view on group agency: what the concept implies, what the literature has said about the concept before, and which methodology the authors are adopting. The authors choose to work from the viewpoint of a "design stance" (p. 13), that is, to identify the limits upon and possibilities for a group to function as an agent, given its structure (its design). The choice allows 
the authors to make extensive use of mathematical tools for analyzing the logical space of group agency: which functions a group can and cannot logically perform, given its composition and decision making design. In what follows I offer a critical summary of the three main sections of the book: "The logical possibility of group agents" (chapters 1 to 3); "The organizational design of group agents" (chapters 4 to 6); and "The normative status of group agents" (chapters 7 to 9).

Chapter 1 of the book provides a "basic account of agency" (p. 19) and agents are defined as those systems possessing the set of three features mentioned above. This first part of the book embeds the assumption of functionalism in List and Pettit's project: Agents are not defined in terms of the material properties they possess, but rather in terms of the functions they perform. While this may be viewed as an innocuous methodological choice, one must note that it begs for justification, something a scrupulous philosopher may expect. The major challenge that arises from the first chapter is how can a group, whose individuals likely hold diverse and possibly conflicting attitudes (beliefs, desires, and the like), form a single and coherent set of such attitudes as required by the definition of group agency?

Chapter 2 takes up the challenge just mentioned and conducts a systematic analysis of the problem of aggregation; most of the results are presented rather informally, and the authors make extensive reference to their previous work on attitude aggregation. The first highlight of this chapter is the fact that a group satisfying four postulated conditions (universal domain, collective rationality, anonymity and systematicity) is logically impossible. This, however, is only of relatively minor interest because the investigation moves beyond the impossibility result to find ways out of the impasse. Group agents are still logically possible, as long as we are willing to relax some of the assumptions. According to List and Pettit, there are very reasonable ways to do that. The authors favour relaxing systematicity as a way of avoiding the impossibility result, and they suggest a premisebased procedure as an optimal aggregation function that would allow a group to transform a multitude of individual attitudes into a collective one. When the group votes on logically interconnected propositions (e.g., A and B, where it holds that "(A \& B) $\rightarrow$ C"), then use of the premise-based procedure implies that agents vote on the premises; consequently, the group arrives at a collective decision by counting the 
votes on the premises, and taking the conclusion that follows logically from those.

Chapter 3 takes up the problem of the logical relation between a group agent and the individuals who are part of it. Is the former reducible to the latter? Or are the two independent? In which sense of independence? List and Pettit show that the relation between a group agent and its parts (the individual agents) is one of supervenience. There are, however, several possible supervenience relations. As in the previous chapter, the authors explore the logical space of the problem and identify those supervenience relations which are most appealing because they satisfy a number of desiderata. As it turns out, group agents in List and Pettit's account are not metaphysically autonomous; but they are autonomous in the epistemological sense: In most cases, it is impossible to reconstruct the individual attitudes of the members of a group starting from the collective attitude of the group itself. In this sense, the supervenience of a group agent on its parts guarantees its epistemological autonomy.

This account of agency, the authors claim, is superior to both reductionist accounts (whereby any attitude attributed to a group is nothing more than a shortcut for attributing it to its component individuals taken singularly), and emergentist ones (according to which the group is something altogether different from its members). It is superior because it justifies the autonomy of a group agent in an unmysterious way (pp. 73-78), that is, without postulating collective objects or collective forces metaphysically subsistent over and above the individual ones.

A critical note to this section is that List and Pettit are perhaps too cautious on the metaphysical implications of defending the supervenient independence of group agents. Suppose we were to compare a "mere collection" (p. 31) to a group agent; while the former, according to the authors' account, is reducible to its individuals without loss of explanation, the latter cannot be explained in the same way. It must be noted however, that a group agent in List and Pettit's sense is not even metaphysically identical with a mere collection. Let us consider a concrete group that would most likely fit the authors' definition of an agent: the Bank of England's Monetary Policy Committee.

The Monetary Policy Committee is in an unmysterious way different from a group of economists meeting at a conference dinner. The Committee's existence is sanctioned by British law, it has a written 
statute, its members meet periodically, deliberate, and vote; moreover, its decisions have a legal status, and so on. In short, the Monetary Policy Committee has a number of features that make it unmysteriously different from the collection of its members (economists and bankers) when they meet at a restaurant for a casual dinner. It is thus reasonable to claim that the group agent considered in this example is not only epistemically independent from the mere collection of its members, but also metaphysically independent, if we consider properties such as 'possessing a statute' or 'possessing a voting mechanism' as legitimate ones. Those properties, to my mind, mark the difference between the group (as an agent) and the mere summation of its parts (the individual agents), and are sufficient for justifying the irreducibility of the Committee to the mere collection of its members.

The second part of the book focuses on specific organizational and design features imposed on a group in order for it to satisfy a number of desiderata. In chapter 4 the desiderata are epistemological. For example, we may impose the desideratum that a group should state the truth of a certain proposition $X$ when $X$ is in fact true, or that it should claim that $X$ is false when $X$ is in fact false. It is important to note that different voting functions satisfy the desiderata to different degrees. Moreover a given voting function may satisfy a certain desideratum to a different degree than it satisfies another. List and Pettit investigate which functions satisfy which desiderata better by use of Bayesian probability theory.

Agents with epistemic desiderata only, however, are of a highly idealized type. In chapters 5 and 6 the authors introduce some more demanding conditions which make the agents they postulate resemble real world agents more closely. In chapter 5 individual agents have more than one dimension of motivations (e.g., truth-seeking and conformism), and can behave strategically. The authors discuss the incentivecompatibility desideratum by means of game theory. In chapter 6 another desideratum is introduced, the control desideratum. For this the authors outline another impossibility result and suggest possible ways out of the impossibility.

The final part of the book takes up the issue of the "normative status of group agents". The question it tries to answer is when and to what extent can groups be held responsible for their actions. Here List and Pettit distinguish between two separate reasons for holding agents responsible for their actions. On the one hand, agents can be held 
responsible instrumentally, that is, in order to regulate their operations and make them criminally liable when they deviate. On the other hand, one may wish to hold an agent responsible in order to create an incentive for that agent to take responsibilities upon him or herself; the authors, borrowing the term from David Garland, call this the practice of "responsibilization". Similarly, List and Pettit argue, a group agent should be held responsible for such formative reasons: that is, to establish a structure of checks and balances in the group, so that no members free-ride on the work of others, or try to hide their personal responsibilities behind that of the collectivity.

But are groups fit to be held responsible? List and Pettit's answer to this question is, in general, positive, at least provided that the groups satisfy those conditions for moral responsibility that are normally imposed on individual agents. That is, when faced with a normatively significant choice, being able to evaluate normative judgments and having the power to act and choose between moral options (p. 158). Elsewhere Pettit has made slightly bolder claims as to the linkage between group agency and group responsibility, claiming that the former entails the latter (see Pettit 2007). In Group agency the authors do not go so far, at least not explicitly, even though it is clear that their three conditions for agency go hand in hand with those for responsibility. A minor remark is in place here. One might wish that the conditions for moral responsibility in this section were argued for to a greater extent; even though it was the choice of the authors to just postulate what seems to be a reasonable account of moral responsibility in three simple conditions, and one that fits well with the conditions for agency postulated earlier in the book.

Aside from those I have discussed, the book covers a number of other topics which do not find space in this review. It should also be noted that the book is hardly a self contained one: Most of the arguments and formal results presented in the text are based on numerous papers previously written by the authors, who often refer the interested reader to those, as well as to the other references in their extensive bibliography.

To conclude, I cannot but warmly recommend this book to anyone who is interested in the specific topics that the book analyzes, but also to anyone who is generally interested in questions of how committees, organizations, and corporations work; what criteria should enter into the design of those groups; and what one should think about when 
trying to understand, and perhaps to improve, the structure and functioning of a group. While one will not find case studies or anything similar to a hands-on manual for corporations in this book, it does provide the all too precious logical structure of the problems which group agency involves; what tools are available to tackle them; and what mind frame one should assume when thinking about a corporation that is meant to function like "the many as one" (see List and Pettit 2005).

\section{REFERENCES}

List, Christian, and Philip Pettit. 2005. On the many as one. Philosophy and Public Affairs, 33 (4): 377-390.

Pettit, Philip. 2007. Responsibility incorporated. Ethics, 117 (2): 171-201.

Carlo Martini is a doctoral student at the Tilburg Center for Logic and Philosophy of Science (TiLPS) at Tilburg University (Netherlands) and lecturer in the Philosophy \& Economics program at Bayreuth University (Germany). His research interests are in the epistemology and philosophy of science, with a particular focus on the philosophy of economics and social epistemology. His current research focuses on the problem of expertise and expert deliberation in economics and on issues of epistemic disagreement and consensus formation in economics and the social sciences.

Contact e-mail: <uni.c.martini@gmail.com>

Website: <http://www.martinicarlo.net/> 